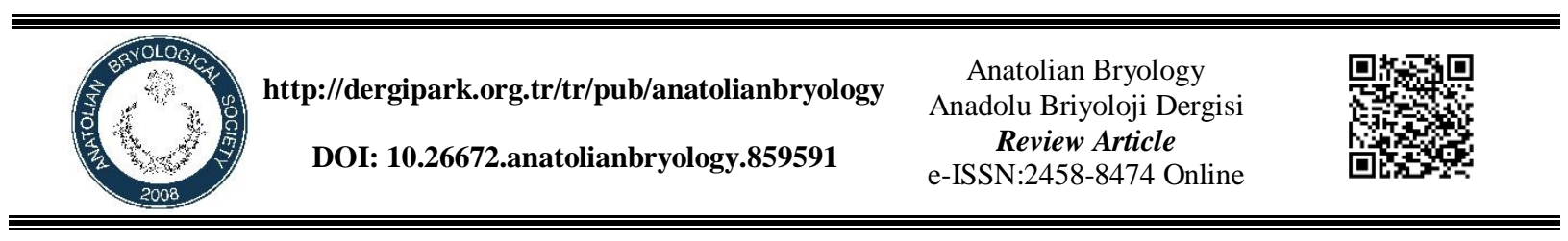

Kastamonu İlinin Ciğerotları ve Boynuzotları Kontrol Listesi

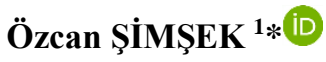

Çanakkale Onsekiz Mart Üniversitesi, Yenice MYO Ormancıllk Bölümü, Çanakkale, TÜRKIYE

\begin{tabular}{lll}
\hline Received: 12 January 2021 & Revised: 18 February 2021 & Accepted: 1 March 2021
\end{tabular}

Öz

$\mathrm{Bu}$ çalışmada; Kastamonu ili sınırları içerisinde gerçekleştirilmiş ciğerotu florası araştırmaları incelenerek, ilin ciğerotu florasının güncel takson listesinin hazırlanması amaçlanmıştır. Buna göre alanda yapılan çalışmaların incelenmesi neticesinde Kastamonu ilinde 1 boynuzotu (Anthoceratophyta) taksonu ile 29 familyaya ait 73 ciğerotu (Marchantiophyta) taksonu bulunduğu tespit edilmiştir. Scapaniaceae familyası 9 takson ile en zengin familya konumundadır.

Anahtar kelimeler: Kastamonu, Flora, Ciğerotları, Marchantiophyta, Boynuzotları, Anthoceratophyta

\title{
The Checklist of Liverworts and Hornworts of Kastamonu Province
}

\begin{abstract}
The aim of this study is to reveal the current taxa list of liverwort flora for the Kastamonu province by examining the previous floristic studies which were done within the boundaries of the province. As a result of assessing the literature data floristic studies, 1 hornwort (Anthoceratophyta) taxon and 73 liverwort (Marchantiophyta) taxa belonging 29 families were listed. The richest Familia is Scapaniaceae with 9 taxa.
\end{abstract}

Keywords: Kastamonu, Flora, Liverworts, Marchantiophyta, Hornworts, Anthoceratophyta

\footnotetext{
* Corresponding author: ozcan.simsek@gmail.com

(C) 2021 All rights reserved / Tüm hakları saklıdır.

To cite this article: Şimşek Ö., 2021. Kastamonu İlinin Ciğerotlarl ve Boynuzotları Kontrol Listesi. Anatolian Bryology. 7:1, 60-69. 


\section{Giriş}

Kastamonu ili Batı Karadeniz bölgesinde $41^{\circ} 21^{\prime}$ kuzey enlemi ile $33^{\circ} 46^{\prime}$ doğu boylamları arasında yer alır. Deniz seviyesinden yüksekliği 775 metredir. Yüzölçümü 13.108,1 km²'dir. $\mathrm{Bu}$, ülke topraklarının \%1,7'sini oluşturur.

Kastamonu ili çoğunlukla engebeli arazilerden oluşmaktadır, ilin kuzeyinde Batı Karadeniz Dağları bulunmaktadır. Karadeniz sahiline paralel olarak İsfendiyar (Küre) Dağları il merkezinin kuzeyinde, güneyinde ise yine doğu batı uzantılı Ilgaz dağları yer alır. Kastamonu'nun yüzölçümünün \%74,6'sı dağlık ve ormanlık alanlardan, \%21,6's1 platolardan ve $\% 3,8$ 'i ovalardan oluşur. (URL 1).

Kastamonu ili, sınırları içinde birçok farklı habitat tipini barındırması nedeniyle tohumlu bitkiler tür çeşitliliği bakımından oldukça zengindir. Avrupa Çevre Ajansı tarafindan belirlenen CORINE (Coordination of Information on the Environment) Çevresel Bilginin Koordinasyonu, Arazi Örtüsü/Kullanımı Sınıflandırmasına göre uydu görüntüleri üzerinden bilgisayar destekli görsel yorumlama metodu ile üretilen arazi örtüsü/kullanımını verisi)'den ve meşcere haritalarından yararlanılarak hazırlanan EUNIS (European Nature Information System) Habitat Tipleri Haritasına göre Kastamonu ilinde 28 farklı habitat tipi görülmektedir (Şekil 1).

Tarım ve Orman Bakanlığı Doğa Koruma ve Milli Parklar Genel Müdürlüğü'nün yürüttüğü 'Ulusal Biyolojik Çeşitlilik Envanter ve İzleme" projesi kapsamında Kastamonu ili için 2019 yılında tamamlanan çalışmaya göre, yapılan literatür taraması ve arazi çalışmaları sonucunda il sınırları içinde toplam 1841 damarlı bitki taksonu tespit edilmiştir. Bunlardan 545'i endemik olup, endemizm oran $1 \% 30$ 'dur (URL 2).

$\mathrm{Bu}$ veriler 1şığında, Kastamonu ilinde tohumsuz bitkilerin de biyolojik çeşitliliğinin yüksek olması muhtemeldir. Dolayısı ile Kastamonu briyofitler konusunda çalışan araştırmacıların ilgisini çeken bir il olmuştur. Çankırı, Ankara, Zonguldak gibi Kastamonu'ya yakın olan illerde briyofitlerle ilgili çalışmalar yürüten araştırmacıların bulunması da bu ilde kapsamlı briyofit florası araştırmaları yapılmasında etkili olmuştur.

Türkiye'de yapılan briyofit flora çalışmaları bölgesel bazda devam etmekle birlikte, henüz tüm ülkeyi kapsayacak düzeye ulaşamamıştır. $\mathrm{Bu}$ nedenle Türkiye'nin ciğerotları florası henüz yazılamamış, çeşitli dönemlerde yayınlanan ulusal ve bölgesel bazdaki kontrol listeleri ile mevcut floristik durumun kayıt altına alınması hedeflenmiştir (Çetin, 1988; Kürschner ve Erdağ 2005; Kiremit ve Keçeli, 2009). Bununla birlikte bölgesel bazda yayınlanan kontrol listelerinin iki farklı anlayışı benimsediği dikkati çekmektedir. Kimi bölgesel kontrol listeleri il bazlı yayınlanmışken (Abay ve ark., 2016; Özdemir ve Batan, 2017a; Özdemir ve Batan, 2017b; Abay ve Ursavaş, 2019), kimi kontrol listeleri Henderson (1961) tarafindan uyarlanan Türkiye kareleme sistemine (Şekil 2) göre düzenlenmiştir (Özdemir, 2009; Abay ve ark., 2010; Ursavaş ve ark., 2010; Keçeli ve ark., 2011). Kastamonu ili Henderson (1961) kareleme sistemine göre A2 karesinde yer almaktadır. 


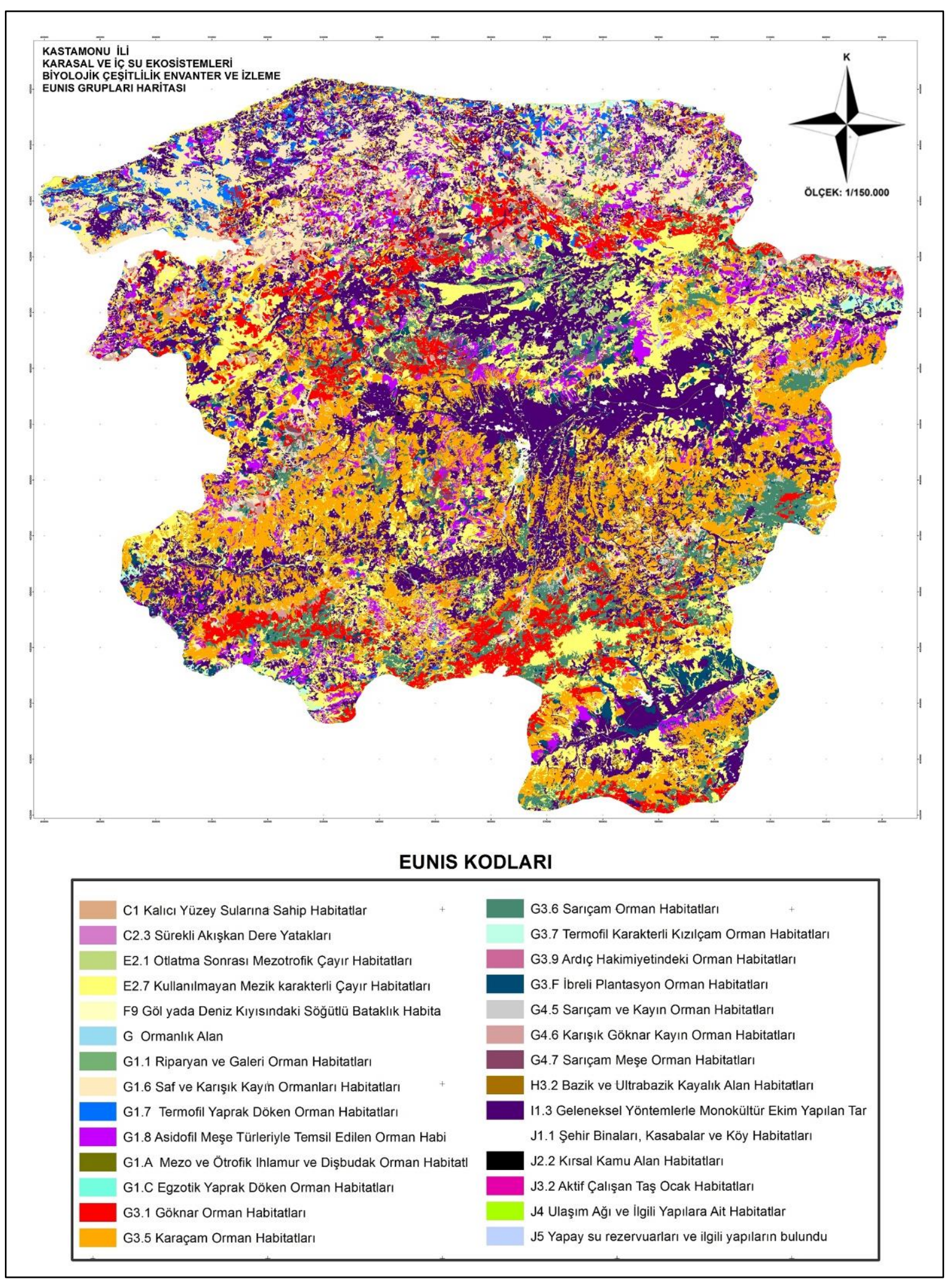

Şekil 1. Kastamonu İli EUNIS Habitat Tipleri Haritası (Tarım ve Orman Bak., 2019) 


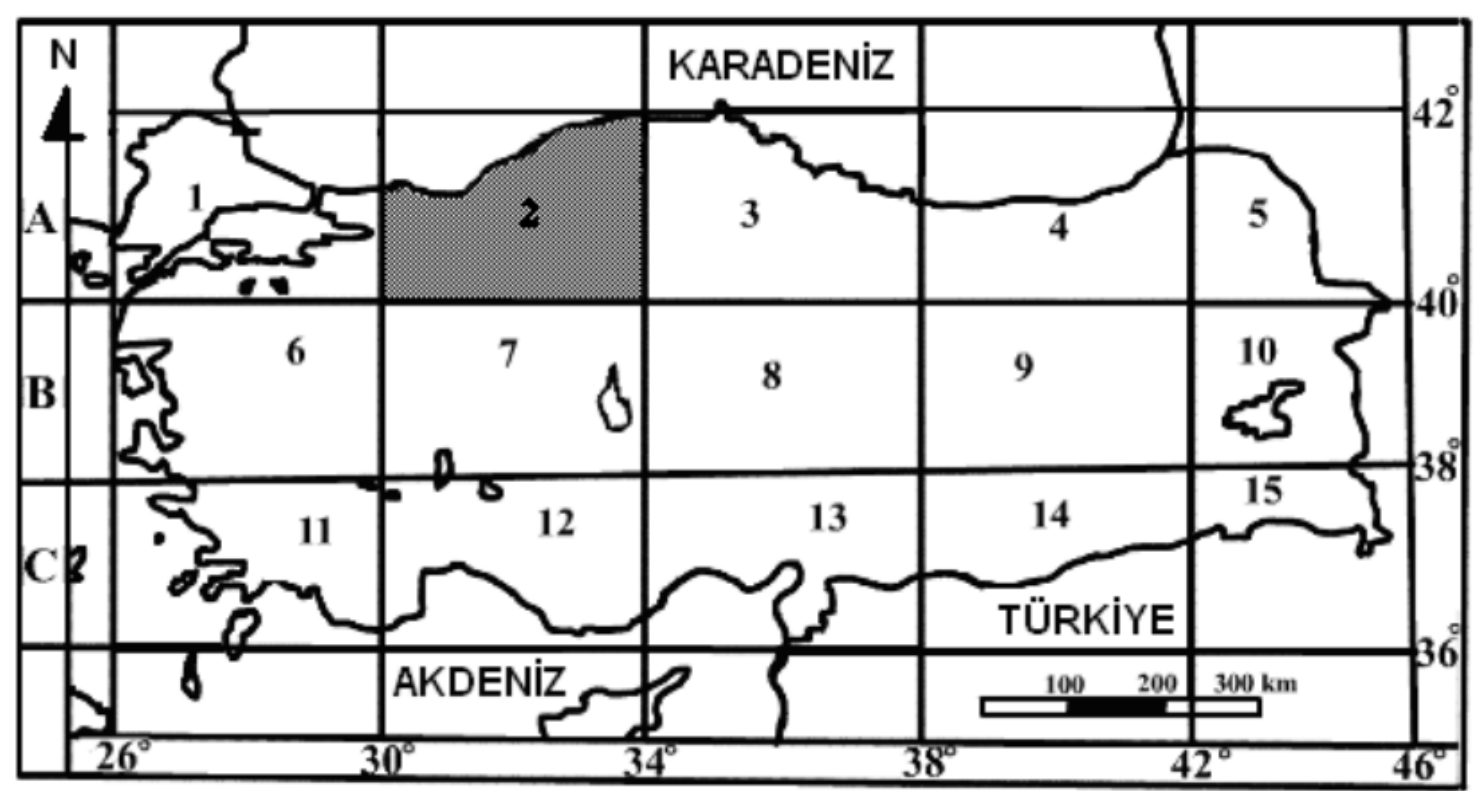

Şekil 2. Henderson (1961) kareleme sistemi

Günümüzde teknolojinin gelişmesine bağlı olarak CBS (Coğrafi Bilgi Sistemleri) konusunda oldukça ilerleme sağlanmış durumdadır. Buna karşılık CBS bu denli ilerleme kaydetmemişken kullanımı geçmişte çeşitli kolaylıklar sağlamış olan Henderson (1961) kareleme sisteminin artık günümüzde araştırmalara kolaylık/pratiklik sağlamadığı düşünülmektedir. $\mathrm{Bu}$ nedenle araştırmalarda Henderson (1961) kareleme sisteminin kullanılmasındansa güncel CBS uygulamalarından yararlanılmasının ve il bazlı ya da bölgesel bazlı kontrol listelerinin artmasının daha faydalı olacağına inanılmaktadır.

$\mathrm{Bu}$ çalışmada yukarıda anlatılan nedenler göz önünde bulundurularak il bazlı kontrol listelerinin hazırlanmasının ileride yapılacak çalışmalar için daha faydalı olacağı düşüncesinden hareketle Kastamonu ilinde bulunan ciğerotlarının kontrol listesinin hazırlanması amaçlanmıştır.

\section{Materyal ve Yöntem}

$\mathrm{Bu}$ çalışmanın materyalini ciğerotu flora çalışmalarının Kastamonu ilini kapsayan literatür verisi oluşturmaktadır. Kontrol listesinin oluşturulması amacıyla Kastamonu ili ve yakın çevresindeki ciğerotlarının araştırıldığı çalışmalar incelenmiş ve Kastamonu il sınırları içinde kalan kayıtlar not edilmiştir. Yapılan literatür taramasında 11 adet yayın tespit edilmiştir. Tespit edilen yayınlar, liste ve harita oluşturulurken kolaylık sağlaması amacıyla kronolojik olarak aşağıdaki gibi numaralandırılmıştır:

Referans 1: Keçeli T. 2004. Batı Karadeniz Bölgesi (Bolu-Zonguldak-Bartın-Kastamonu)
Ciğerotları (Hepaticae) Florası. Ankara Üniversitesi Fen Bilimleri Enstitüsü. Doktora Tezi.

Referans 2: Keçeli T. Çetin B. Uyar G. 2004. Riccardia latifrons (Lindb.) Lindb. in New national and regional bryophyte records, 9. Journal of Bryology. 26, 63-66

Referans 3: Keçeli T. Çetin B. 2006. A contribution to the liverwort flora of Western Black Sea Region, northern Turkey, and a new record (Cephaloziella dentata, Cephaloziellaceae) to Southwest Asia. Cryptogamie, Bryologie. 27, 459-470.

Referans 4: Ursavaş S. Abay G. 2009. Contributions to the bryoflora of Ilgaz Mountains, Yenice Forests, Turkey. Biological Diversity and Conservation. 2:3, 112-121.

Referans 5: Şimşek Ö. Çetin B. Canlı K. 2011. Contributions to the Liverwort (Marchantiophyta) flora of Ilgaz Mountains (Turkey). Biological Diversity and Conservation. 4:1, 7-10.

Referans 6: Keçeli T. Ören M. Uyar G. 2012. Türkiye Ciğerotları (Marchantiophyta) Florası için İki Yeni Kayıt: Cephalozia lunulifolia (Cephaloziaceae) ve Lophocolea fragrans (Lophocoleaceae). 21. Ulusal Biyoloji Kongresi, 03-07 Eylül 2012, Ege Üniversitesi, İzmir, Türkiye. Poster bildiri.

Referans 7: Ören M. Uyar G. Keçeli T. 2012. The bryophyte flora of the western part of the Küre Mountains (Bartın, Kastamonu), Turkey. Turkish Journal of Botany, 36:5, 538-557. 
Referans 8: Şimşek Ö. Çetin B. Canlı K. 2014. Pressia quadrata (Scop.) NEES (Marchantiaceae), new to the liverwort flora of Turkey. Phytologia Balcanica. 20, 155157.

Referans 9: Ören, M., Sarı, B., Ursavaş, S. (2015). Syntrichia minor (Pottiaceae) and Cephaloziella integerrima (Cephaloziellaceae) new to bryophyte flora of Turkey. Archives of Biological Sciences, 67(2), 367-372.

Referans 10: Ören M. Ursavaş S. 2020. Doğu Küre Dağları Briyofit Florası. Anatolian Bryology. $6: 2,78-96$

Referans 11: Unan A.D. Potemkin A. Ursavaş S. Çalışkan S. Ören M. 2020. New records of two Scapania species (Scapaniaceae, Marchantiophyta) from north of Turkey, Plant Biosystems - An International Journal
Dealing with all Aspects of Plant Biology. DOI: 10.1080/11263504.2020.1779836.

Taksonların geçerli isimleri, sinonimler ve listenin oluşturulmasında Hodgetts ve ark. (2020)'den yararlanılmıştır. Bunların dışında dünya ciğerotları ve boynuzotları kontrol listesinden de (Söderström ve ark., 2016) bu konuda destek alınmıştır. Kontrol listesinden yararlanacak okuyuculara kolaylık sağlaması amaciyla liste takson isimlerinin alfabetik sıralamasına göre hazırlanmıştır.

\section{Bulgular}

Elde edilen bulgulara göre Kastamonu ilinde 1 boynuzotu taksonu ve 29 familyaya ait 73 ciğerotu taksonu bulunduğu tespit edilmiştir (Tablo 1). Bunlardan Scapaniaceae familyası 9 takson ve $\% 12,33$ 'lik oran ile en zengin familya olarak dikkati çekmektedir (Tablo 2).

Tablo 1. Tür listesi

\section{TÜR}

\begin{tabular}{|c|c|c|}
\hline & & \\
\hline Anthoceros punctatus L.* & Anthocerotaceae* & 7,10 \\
\hline Aneura pinguis (L.) Dum. & Aneuraceae & $5,7,10$ \\
\hline $\begin{array}{l}\text { Apopellia endiviifolia (Dicks.) Nebel \& D.Quandt } \\
\text { [Syn: Pellia endiviifolia (Dicks.) Dumort] }\end{array}$ & Pelliaceae & $1,3,7,10$ \\
\hline Barbilophozia barbata (Schmidel ex Schreb.) Loeske & Anastrophyllaceae & $1,3,7,10$ \\
\hline Barbilophozia hatcheri (A.Evans.) Loeske & Anastrophyllaceae & $4,5,10$ \\
\hline Blasia pusilla $\mathrm{L}$ & Blasiaceae & 10 \\
\hline Blepharostoma trichophyllum (L.) Dumort. & Blepharostomataceae & 10 \\
\hline Calypogeia arguta Nees \& Mont. & Calypogeiaceae & 5 \\
\hline Calypogeia fissa (L.) Raddi & Calypogeiaceae & $1,7,10$ \\
\hline Cephalozia bicuspidata (L.) Dumort. & Cephaloziaceae & 7,10 \\
\hline Cephaloziella divaricata (Sm.) Schiffn. & Cephaloziellaceae & 7,10 \\
\hline Cephaloziella integerrima (Lindb.) Warnst. & Cephaloziellaceae & 9 \\
\hline Cephaloziella rubella (Nees) Warnst. & Cephaloziellaceae & 7,10 \\
\hline Cephaloziella stellulifera Schiffin. & Cephaloziellaceae & 5 \\
\hline Cephaloziella turneri (Hook.) Müll.Frib. & Cephaloziellaceae & 7 \\
\hline Chiloscyphus pallescens (Ehrh. ex Hoffm.) Dumort. & Lophocoleaceae & $1,3,4$ \\
\hline Chiloscyphus polyanthos (L.) Corda & Lophocoleaceae & $1,7,10$ \\
\hline Cololejeunea rosettiana (C. Massal.) Schiffn. & Lejeuneaceae & 1,7 \\
\hline Conocephalum conicum (L.) & Conocephalaceae & $1,5,7,10$ \\
\hline Diplophyllum albicans (L.) Dumort. & Scapaniaceae & $1,7,10$ \\
\hline Fossombronia angulosa (Dicks.) Raddi & Fossombroniaceae & 10 \\
\hline $\begin{array}{l}\text { Fossombronia caespitiformis De Not. Ex Rabenh. subsp. } \\
\text { multispira (Schiff n.) J.R.Bray and D.C.Cargill }\end{array}$ & Fossombroniaceae & 7 \\
\hline Frullania dilatata $(\mathrm{L}$.$) Dum.$ & Frullaniaceae & $1,5,7,10$ \\
\hline Frullania tamarisci (L.) Dumort. & Frullaniaceae & $1,7,10$ \\
\hline Fuscocephaloziopsis lunulifolia (Dumort.) Váňa \& L.Söderstr. & Cephaloziaceae & 6,10 \\
\hline $\begin{array}{l}\text { Jubula hutchinsiae (Hook.) Dumort. subsp. caucasica Konstant. } \\
\text { \& Vilnet } * *\end{array}$ & Jubulaceae & 1,7 \\
\hline Jungermannia atrovirens Dum. & Jungermanniaceae & 3,5 \\
\hline Lejeunea cavifolia (Ehrh.) Lindb. & Lejeuneaceae & $1,7,10$ \\
\hline Lepidozia reptans (L.) Dumort. & Lepidoziaceae & 10 \\
\hline $\begin{array}{l}\text { Liochlaena lanceolata Nees } \\
\text { [Syn: Jungermannia leiantha Grolle] }\end{array}$ & Jungermanniaceae & 10 \\
\hline
\end{tabular}




\begin{tabular}{|c|c|c|}
\hline TÜR & FAMILYY & REF. No \\
\hline Lophocolea minor Nees & Lophocoleaceae & $1,3,4,5,7,10$ \\
\hline Lophocolea bidentata (L.) Dumort. & Lophocoleaceae & $1,5,7,10$ \\
\hline Lophocolea heterophylla (Schrad.) Dum. & Lophocoleaceae & $1,3,5,7,10$ \\
\hline Lophozia ventricosa (Dicks.) Dum. & Lophoziaceae & $1,3,5,10$ \\
\hline Lunularia cruciata (L.) Dumort. ex Lindb. & Lunulariaceae & $3,5,7,10$ \\
\hline Marchantia polymorpha $\mathrm{L}$. & Marchantiaceae & $1,3,4,5,7$ \\
\hline Marchantia polymorpha L. subsp. montivagans & Marchantiaceae & 10 \\
\hline $\begin{array}{l}\text { Marchantia quadrata Scop. } \\
\text { [Syn: Preissia quadrata (Scop.) Nees] }\end{array}$ & Marchantiaceae & 8 \\
\hline Mesoptychia bantriensis (Hook.) L.Söderstr. \& Váňa & Jungermanniaceae & 10 \\
\hline $\begin{array}{l}\text { Mesoptychia collaris (Nees) L.Söderstr. \& Váňa } \\
\text { [Syn: Leiocolea alpestris (Schleich. ex F. Weber) Isov.] }\end{array}$ & Jungermanniaceae & 10 \\
\hline $\begin{array}{l}\text { Mesoptychia turbinata (Raddi) L.Söderstr. \& Váňa } \\
\text { [Syn= Leiocolea turbinata (Raddi) H.Buch] }\end{array}$ & Jungermanniaceae & 7,10 \\
\hline Metzgeria conjugata Lindb. & Metzgeriaceae & $1,7,10$ \\
\hline $\begin{array}{l}\text { Metzgeria furcata (L.) Dumort. } \\
\text { [Syn: Metzgeria furcata (L.) Dumort. var ulvula] }\end{array}$ & Metzgeriaceae & $1,3,5,7,10$ \\
\hline $\begin{array}{l}\text { Metzgeria pubescens (Schrank) Raddi } \\
\text { [Syn: Apometzgeria pubescens (Schrank) Kuwah.] }\end{array}$ & Metzgeriaceae & $1,3,7$ \\
\hline Nowellia curvifolia (Dicks.) Mitt & Cephaloziaceae & 7 \\
\hline Pedinophyllum interruptum (Nees) Kaal. & Plagiochilaceae & $1,7,10$ \\
\hline Pellia epiphylla (L.) Corda & Pelliaceae & $1,3,4,5$ \\
\hline Plagiochila asplenioides (L. emend. Taylor) Dumort. & Plagiochilaceae & $1,7,10$ \\
\hline Plagiochila porelloides (Torrey ex Nees) Lindenb. & Plagiochilaceae & $1,3,4,5,7,10$ \\
\hline Porella arboris-vitae (With.) Grolle & Porellaceae & 1,10 \\
\hline Porella cordaeana (Huebener) Moore & Porellaceae & $1,4,7$ \\
\hline Porella obtusata (Tayl.) Trev. & Porellaceae & 5 \\
\hline Porella pinnata $\mathrm{L}$. & Porellaceae & 4 \\
\hline Porella platyphylla (L.) Pfeiff. & Porellaceae & $1,5,7,10$ \\
\hline Ptilidium pulcherrimum (Weber) Vain. & Ptilidiaceae & $1,7,10$ \\
\hline Radula complanata (L.) Dumort. & Radulaceae & $1,4,5,7,10$ \\
\hline Radula lindenbergiana Gottsche ex C. Hartm. & Radulaceae & $1,5,7,10$ \\
\hline Reboulia hemisphaerica (L.) Raddi & Aytoniaceae & 10 \\
\hline Riccardia chamedryfolia (With.) Grolle & Aneuraceae & 7,10 \\
\hline Riccardia latifrons (Lindb.) Lindb. & Aneuraceae & 1,2 \\
\hline Riccardia palmata (Hedw.) Carruth. & Aneuraceae & 5,10 \\
\hline Riccia gougetiana & Ricciaceae & 10 \\
\hline Riccia sorocarpa Bisch. & Ricciaceae & 10 \\
\hline Scapania aequiloba (Schwägr.) Dumort. & Scapaniaceae & $1,3,7,10$ \\
\hline Scapania aspera Bernet et M. Bernet & Scapaniaceae & $1,3,7$ \\
\hline Scapania glaucocephala (Taylor) Austin & Scapaniaceae & 11 \\
\hline Scapania irrigua (Nees) Nees & Scapaniaceae & $1,3,5,7$ \\
\hline Scapania nemorea (L.) Grolle & Scapaniaceae & 5 \\
\hline Scapania umbrosa (Schrad.) Dumort & Scapaniaceae & 10 \\
\hline Scapania undulata (L.) Dumort. & Scapaniaceae & $1,3,10$ \\
\hline Scapania verrucosa Heeg & Scapaniaceae & 1,3 \\
\hline Solenostoma gracillimum (Sm.) R.M.Schust. & Solenostomataceae & 10 \\
\hline Southbya tophacea (Spruce) Spruce & Southbyaceae & 1,7 \\
\hline $\begin{array}{l}\text { Trilophozia quinquedentata (Huds.) Bakalin } \\
\text { [Syn=Tritomaria quinquedentata (Huds.) H.Buch] }\end{array}$ & Lophoziaceae & 1,3 \\
\hline
\end{tabular}

* Boynuzotu (Anthocerotophyta) taksonu

** Referans 1 (Keçeli, 2004)'de Jubula javanica Steph. olarak verilen takson. Jubula hutchinsiae (Hook.) Dumort. subsp. javanica (Steph.) Verd. taksonunun sinonimidir. Referans 1 (Keçeli, 2004) ve Referans 7 (Ören ve ark., 2012)'de bildirilmiş olan bu taksonun Konstantinova ve Vilnet (2011) ile Kürschner (2013) tarafindan yapılan çalışmalarla Türkiye'de kayda geçmiş örneklerinin Jubula hutchinsiae (Hook.) Dumort. subsp. caucasica Konstant. \& Vilnet taksonuna ait olduğunu bildirmiştir. 
Tablo 2. Familyalara ait takson sayıları ve oranları

\begin{tabular}{llr|llr} 
Familya & $\begin{array}{l}\text { Takson } \\
\text { Sayısı }\end{array}$ & Oranı (\%) & Familya & $\begin{array}{l}\text { Takson } \\
\text { Sayıs }\end{array}$ & $\begin{array}{r}\text { Oranı } \\
(\mathbf{\%})\end{array}$ \\
\hline Scapaniaceae & 9 & 12,33 & Lophoziaceae & 2 & 2,74 \\
\hline Cephaloziellaceae & 5 & 6,85 & Pelliaceae & 2 & 2,74 \\
\hline Jungermanniaceae & 5 & 6,85 & Radulaceae & 2 & 2,74 \\
\hline Lophocoleaceae & 5 & 6,85 & Ricciaceae & 2 & 2,74 \\
\hline Porellaceae & 5 & 6,85 & Aytoniaceae & 1 & 1,37 \\
\hline Aneuraceae & 4 & 5,48 & Blasiaceae & 1 & 1,37 \\
\hline Cephaloziaceae & 3 & 4,11 & Blepharostomataceae & 1 & 1,37 \\
\hline Marchantiaceae & 3 & 4,11 & Conocephalaceae & 1 & 1,37 \\
\hline Metzgeriaceae & 3 & 4,11 & Jubulaceae & 1 & 1,37 \\
\hline Plagiochilaceae & 3 & 4,11 & Lepidoziaceae & 1 & 1,37 \\
\hline Anastrophyllaceae & 2 & 2,74 & Lunulariaceae & 1 & 1,37 \\
\hline Calypogeiaceae & 2 & 2,74 & Ptilidiaceae & 1 & 1,37 \\
\hline Fossombroniaceae & 2 & 2,74 & Solenostomataceae & 1 & 1,37 \\
\hline Frullaniaceae & 2 & 2,74 & Southbyaceae & 1 & 1,37 \\
\hline Lejeuneaceae & 2 & 2,74 & TOPLAM & $\mathbf{7 3}$ & $\mathbf{1 0 0 , 0 0}$ \\
\hline
\end{tabular}

CBS (Coğrafi Bilgi Sistemleri) uygulamalarından yararlanılarak Kastamonu ilinde ciğerotu örneklerinin tespit edildiği noktaların koordinatları incelenmiș ve neticesinde bir harita hazırlanmıștır (Şekil 3). Ilgaz dağları briyoflorası ile ilgili bir çalışma olan Referans 4 (Ursavaş ve Abay, 2009)'te çalışma alanı Çankırı ili Yenice ormanları olarak belirtilmiş olmakla birlikte ilgili çalışmada koordinatları verilen 44 istasyondan $25,27,28$ ve 29 no'lu istasyonların Kastamonu il sınırları içinde kaldığı görülmüss, bu nedenle bu istasyonlardaki taksonlar kontrol listesine, ilgili lokaliteler de haritaya dahil edilmiştir. Yine Referans 5'te (Şimşek ve ark., 2011) Ilgaz dağlarından bildirilen 6 istasyondan $1,2,3,4$ ve 5 no'lu istasyonlar Kastamonu il sınırları içinde kaldığından bu lokaliteler haritaya ve bu lokalitelerden bildirilen taksonlar da kontrol listesine dahil edilmiștir. Diğer tüm referanslar ile ilgili benzer çalışma yapılıış ve çalışmalarda bildirilen koordinatlardan
Kastamonu il sınırları içinde kalan lokaliteler haritada gösterilmiştir. Yalnızca Referans 3'te (Keçeli ve Çetin, 2006) istasyonlarla ilgili koordinat bilgisi verilmeyip yer tarifi şeklinde lokalite bildirildiğinden bahse konu haritada Referans 3'e ait gösterim bulunmamaktadır.

Hazırlanan harita ile çalışmaların hangi bölgelerde yoğunlaştığı ve hangi bölgelerde çalışma yapılmasına ihtiyaç olduğu gibi sorulara cevap aranmıştır. Buna göre Kastamonu ilinin kuzey kesiminde kalan Batı Küre Dağları ile Doğu Küre Dağlarında daha kapsamlı ve detaylı çalışmalar yapıldığı, güneyde yer alan Ilgaz Dağları ve civarında daha küçük çaplı araştırmalar yapıldığı görülmektedir. Ayrıca İhsangazi ve Seydiler ilçelerinden hiç örnekleme yapılmadığı, Araç, Tosya, Taşköprü ve Kastamonu Merkez ilçelerinde ise çok az örnekleme yapıldığı anlaşılmaktadır. 


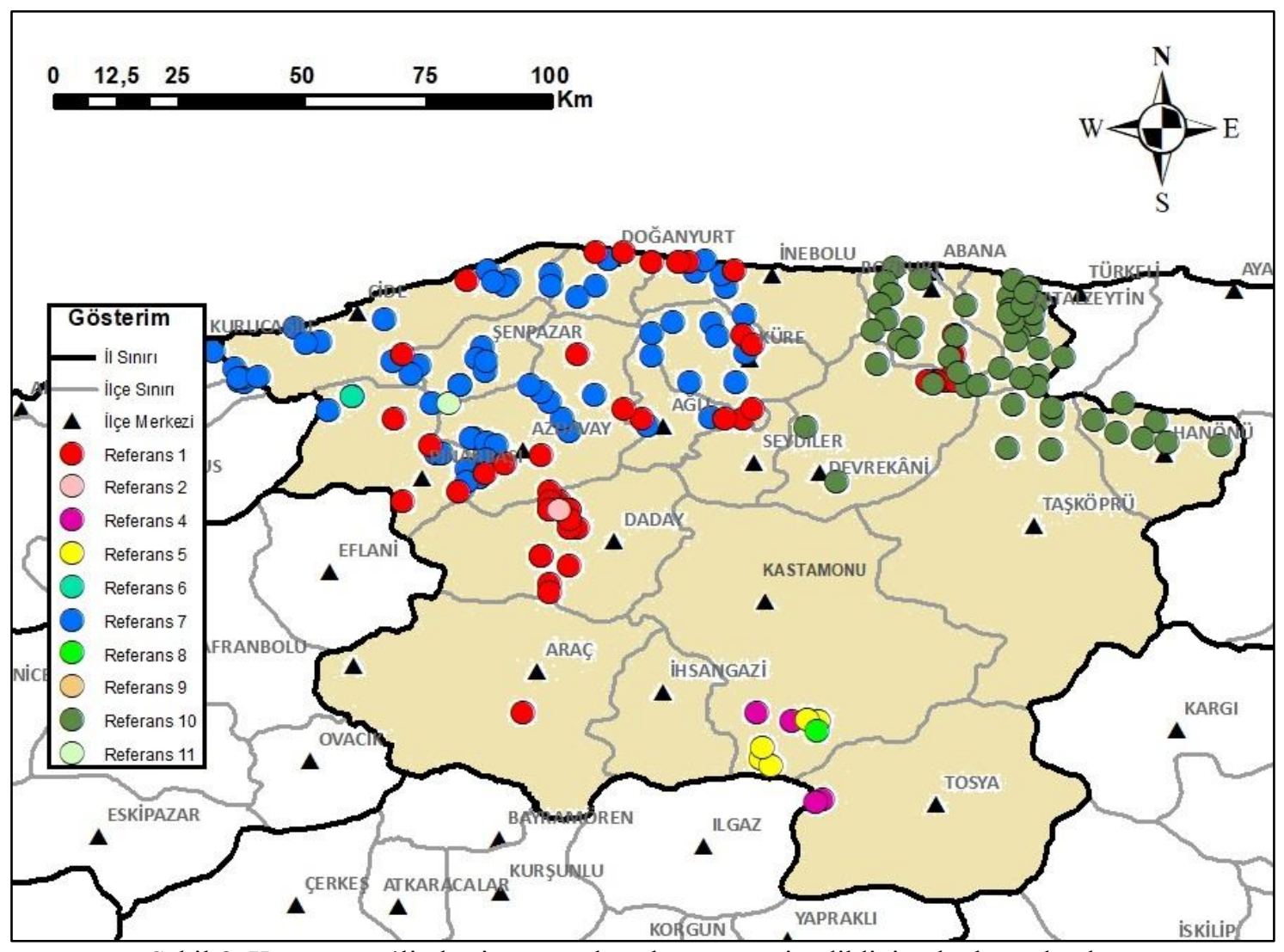

Şekil 3. Kastamonu ilinde ciğerotu taksonlarının tespit edildiği noktaların dağılımı

\section{Tartışma ve Sonuç}

Yapılan bu çalışma ile Kastamonu ilinde ciğerotu florası üzerine yapılmış olan araştırmalar derlenmiş ve Kastamonu ilinin ciğerotları kontrol listesi hazırlanmışıtır. Bununla birlikte yapılmış olan çalışmalarda Kastamonu il sınırları içinde ciğerotu örneklerinin toplandığı koordinatların haritası hazırlanmışıtır.

Çalışma neticesinde Kastamonu ilinde 1 boynuzotu taksonu ve 29 familyaya ait 73 ciğerotu taksonu bulunduğu tespit edilmiştir. Bunlardan Scapaniaceae familyası 9 takson ve $\% 12,33$ 'lik oran ile en zengin familyadır.

Kastamonu'ya komşu olan Çankırı ilinde 37 ciğerotu taksonu bulunurken (Abay ve Ursavaş, 2019) Rize'de 82 ciğerotu (Abay ve ark., 2016), 1 boynuzotu taksonu, Giresun'da 17 ciğerotu taksonu (Özdemir ve Batan, 2017a), Trabzon'da 49 ciğerotu ve 1 boynuzotu taksonu (Özdemir ve Batan, 2017b) tespit edilmiştir. Bu rakamlarla karşılaştıııldığında Kastamonu ilinin ciğerotları florasının zengin bir tür çeşitliliği barındırdığından bahsedilebilir.

Bunun yanında Kastamonu ilinden ciğerotu toplanan noktaların koordinatları harita üzerinde incelendiğinde çalışmaların ilin kuzeyinde yer alan
Batı ve Doğu Küre Dağları ile yakın çevresinde daha kapsamlı olarak yapıldığı, Ilgaz Dağlarında ise daha fazla araştırma yapılmasına ihtiyaç olduğu görülmektedir. Bununla birlikte İhsangazi ve Seydiler ilçelerinde hiç örnekleme yapılmadığı, Araç, Tosya, Taşköprü ve Kastamonu Merkez ilçelerinde çok az örnekleme yapıldığı dikkati çekmektedir. İleride Kastamonu ilinde yapılacak çalışmalarda bu bölgelerin araştırma alanı olarak seçilmesi ilin briyofloristik çeşitlilik potansiyelinin tam anlamı ile ortaya çıkarılmasında önemli rol oynayacaktır. Tüm bu hususlar neticesinde Kastamonu ilinde çeşitli briyofloristik çalışmalar yapılmış olmasına rağmen, halen ciğerotu florasının araştırılması gereken birçok alan olduğu ortaya çıkmaktadır.

Gerek Kastamonu'da gerekse ülkenin diğer illerinde briyofitlerle ilgili yapılacak olan flora araştırmalarında CBS (Coğrafi Bilgi sistemleri) uygulamalarından daha fazla yararlanılması gerektiğine inanılmaktadır. $\mathrm{Bu}$ sayede detaylı haritalar ve veri tabanları hazırlanmasına olanak sağlanabilecektir. Detaylı haritalar yapılması, CBS'ne uygun veri tabanları üretilmesi ile ülkemizdeki briyofloristik çalışmalar bir adım ileriye taşınarak, taksonların bölgesel ve ulusal düzeyde yayılış alanları, popülasyon yoğunlukları, popülasyonların alansal büyüklükleri gibi veriler 
üretilebilecektir. Böylelikle taksonların nesillerinin tehdit durumları (IUCN kriterleri), nadirlik, endemiklik gibi çeşitli özellikleri daha iyi anlaşılabilecektir. Ayrıca tür ve popülasyon düzeyinde biyolojik çeşitlilik izleme çalışmaları yapilabilecektir. Biyologlar ve orman mühendisleri başta olmak üzere arazi çalışması yapan ve flora araştırmaları yürüten meslek mensuplarının güncel teknolojik ilerlemelerden daha iyi faydalanabilmesi, daha verimli ve daha nitelikli çalışmalar yapabilmesi için hem lisans hem lisansüstü seviyede CBS eğitimleri almaları bu konuda ilerleme kaydedilmesine katk1 sağlayacaktır.

\section{Kaynaklar}

Abay G. Ursavaş S. Keçeli T. 2010. Türkiye'nin B7 karesinin bryophyta kontrol listesi, 20. Ulusal Biyoloji Kongresi, Denizli, Türkiye. 21-25 Haziran 2010. s. 394-395.

Abay G. Ursavaş S. 2019. Çankırı İlinin Briyofit Listesi. Anatolian Bryology. 5:1, 56-64.

Abay G. Batan N. Özdemir T. 2016. Bryophyte checklist of Rize, North-East Turkey. Arctoa. 25, 386-392.

Çetin B. 1988. Checklist of liverworts and hornworts of Turkey, Lindbergia. 14, 1214.

Henderson, DM. 1961. Contribution to bryophyte flora of Turkey IV. Notes of the Royal Botanical Garden Edinburgh. 23, 263-278.

Hodgetts N.G. 2015. Checklist and country status of European bryophytes - toward a new Red List of Europe, Irish Wildlife Manuals. 84, 1-125.

Hodgetts N.G. Söderström L. Blockeel T.L. Caspari S. Ignatov M.S. Konstantinova N.A. Lockhart N. Papp B. Schröck C. SimSim M. ve ark. 2020. An annotated checklist of bryophytes of Europe, Macaronesia and Cyprus. Journal of Bryology. 42: 1, 1-116.

Keçeli T. Ursavaş S. Abay G. 2011. Türkiye'nin B6 karesinin bryophyta kontrol listesi, Bartın Orman Fakültesi Dergisi. 13, 14-24.

Keçeli T. 2004. Batı Karadeniz Bölgesi (BoluZonguldak-Bartın-Kastamonu) Ciğerotları (Hepaticae) Florası. Ankara Üniversitesi Fen Bilimleri Enstitüsü. Doktora Tezi.

Keçeli T. Çetin, B. 2006. A contribution to the liverwort flora of Western Black Sea Region, northern Turkey, and a new record (Cephaloziella dentata, Cephaloziellaceae) to Southwest Asia. Cryptogamie, Bryologie. 27, 459-470.

Keçeli T. Çetin B. Uyar G. 2004. Riccardia latifrons (Lindb.) Lindb. in New national and regional bryophyte records, 9. Journal of Bryology. 26, 63-66.

Keçeli T. Ören M. Uyar G. 2012. Türkiye Ciğerotları (Marchantiophyta) Florası için İki Yeni Kayıt: Cephalozia lunulifolia (Cephaloziaceae) ve Lophocolea fragrans (Lophocoleaceae). 21. Ulusal Biyoloji Kongresi, 03-07 Eylül 2012, Ege Üniversitesi, İzmir, Türkiye. Poster bildiri.

Kiremit H.Ö. ve Keçeli T. 2009. An Annotated Check-list of the Hepaticae and Anthocerotae of Turkey, Cryptogamie Bryologie. 30:3, 343-356.

Konstantinova N.A. Vilnet A.A. 2011. Jubula hutchinsiae subsp. caucasica subsp. nov. Jubulaceae, Marchantiophyta) - a new taxon from the western Caucasus. Arctoa. 20, 227-238.

Kürschner H. Frey W. 2011. Liverworts, mosses and hornworts of Southwest Asia (Marchantiophyta, Bryophyta, Anthocerotophyta), Nova Hedwigia, Beiheft. 139, 1-240.

Kürschner H. 2013. Jubula hutchinsiae subsp. caucasica Konstant.\& Vilnet (Marchantiophyta: Jubulaceae - new for the bryophyte flora of Iran and Turkey. Polish Botanical Journal. 58, 211-216.

Kürschner H. Erdağ A. 2005. Bryophytes of Turkey: An Annotated Reference List of the Species with Synonymus from the Recent Literature and an Annotated List of Turkish Bryological Literature. Turkish Journal of Botany. 29, 95-154.

Ören M. Ursavaş S. 2020. The Bryophyte Flora of Doğu Küre Mountains. Anatolian Bryology. 6:2, 78-96.

Ören M. Uyar G. Keçeli T. 2012. The bryophyte flora of the western part of the Küre Mountains (Bartın, Kastamonu), Turkey. Turkish Journal of Botany. 36:5, 538-557.

Ören M. Sarı B. Ursavaş S. 2015. Syntrichia minor (Pottiaceae) and Cephaloziella integerrima (Cephaloziellaceae) new to bryophyte flora of Turkey. Archives of Biological Sciences. 67:2, 367-372.

Özdemir T. Batan N. 2017a. Bryophyte Checklist of Giresun, North East Turkey. Anatolian Bryology. 3:1, 1-8.

Özdemir T. Batan N. 2017b. The bryophyte checklist of Trabzon province of Turkey. Arctoa. 26, 58-67.

Özdemir T. 2009. A revised checklist of Bryophytes of A4 square of Turkey, International Journal of Botany. 5, 1-35.

Ros R.M. Mazımpaka V. Abou-Salama U. Aleffi M. Blockeel TL. Brugués M. Cano M.J. Cros RM. Dia M.G. Dirkse G.M. El 
Saadawi W. Erdağ A. Ganeva A. GonzálezMancebo J.M. Herrnstadt I. Khalıl K. Kürschner H. Lanfranco E. Losada-Lima A. Refa1 M.S. Rodriguez- Nuñez S. Sabovljević M. Sérgio C. Shabbara H. SimSim M. Söderström L. 2007. Hepatics and Anthocerotes of the Mediterranean, an annotated checklist, Cryptogamie, Bryologie. 28:4, 351- 437.

Söderström, L. Hagborg, A. von Konrat, M. Bartholomew-Began, S. Bell D. Briscoe L. Brown E. Cargill D.C. Costa D.P. CrandallStotler B.J. ve ark. 2016. World checklist of hornworts and liverworts. PhytoKeys. 59, $1-828$.

Şimşek Ö. Çetin B. Canlı K. 2011. Contributions to the Liverwort (Marchantiophyta) flora of Ilgaz Mountains (Turkey). Biological Diversity and Conservation. 4:1, 7-10.

Şimşek Ö. Çetin B. Canlı K. 2014. Pressia quadrata (Scop.) NEES(Marchantiaceae), new to the liverwort flora of Turkey. Phytologia Balcanica. 20, 155-157.

Tarım ve Orman Bakanlığı. 2019. Kastamonu İli'nin Karasal ve İç Su Ekosistemleri
Biyolojik Çeşitlilik Envanter ve İzleme İşi Sonuç Raporu. Kastamonu.

Unan A.D. Potemkin A. Ursavaş S. Çalışkan S. Ören M. 2020. New records of two Scapania species (Scapaniaceae, Marchantiophyta) from north of Turkey, Plant Biosystems - An International Journal Dealing with all Aspects of Plant Biology. DOI: 10.1080/11263504.2020.1779836.

URL 1. Kastamonu Valiliği. Website: http://www.kastamonu.gov.tr/cografi-yapisi [Erişim: 16.02.2021].

URL 2. Nuh'un Gemisi Ulusal Biyolojik Çeşitlilik Veritaban1. 2021. Website: http://www.nuhungemisi.gov.tr [Erişim:12.01.2021].

Ursavaş S. Keçeli T. Abay G. 2010. Türkiye'nin B8 karesinin bryophyta kontrol listesi, 20. Ulusal Biyoloji Kongresi, 21 - 25 Haziran 2010. 134, 392-393.

Ursavaş S. Abay G. 2009. Contributions to the bryoflora of Ilgaz Mountains, Yenice Forests, Turkey. Biological Diversity and Conservation. $\quad 2: 3, \quad 112-121$. 